\title{
Interaction dynamics of electrostatic solitary waves
}

\author{
V. L. Krasovsky', H. Matsumoto ${ }^{2}$, and Y. Omura ${ }^{2}$ \\ ${ }^{1}$ Space Research Institute. Russian Academy of Sciences, Profsoyuznaya 84/32, Moscow 117810, Russia \\ ${ }^{2}$ Radio Atmospheric Science Center, Kyoto University, Uji. Kyoto 611-0011, Japan
}

Received: 8 June 1999 - Accepted: 13 September 1999

\begin{abstract}
Interaction of nonlinear electrostatic pulses associated with electron phase density holes moving in a collisionless plasma is studied. An elementary event of the interaction is analyzed on the basis of the energy balance in the system consisting of two electrostatic solitary waves. It is established that an intrinsic property of the system is a specific irreversibility caused by a nonadiabatic modification of the internal structure of the holes and their effective heating in the process of the interaction. This dynamical irreversibility is closely connected with phase mixing of the trapped electrons comprising the holes and oscillating in the varying self-consistent potential wells. As a consequence of the irreversibility, the "collisions" of the solitary waves should be treated as "inelastic" ones. This explains the general tendency to the merging of the phase density holes frequently observed in numerical simulation and to corresponding coupling of the solitary waves.
\end{abstract}

\section{Introduction}

Waveform observations performed by Plasma Wave Instrument aboard the Geotail spacecraft have provided rather unexpected data on the high-frequency component of broadband electrostatic noise in the distant magnetotail (Matsumoto et al., 1994). It appears that the corresponding wave perturbations present bipolar electric field spikes which may be treated as unipolar pulses of the electrostatic potential moving along the magnetotail (Omura et al., 1994). These perturbations were called electrostatic solitary waves (ESW) due to their polarization and typical isolated wave signatures. The observed waveforms are difficult to explain within the framework of the linear plasma wave theory. This has led Matsumoto et al. (1994) to presume that the revealed wave perturbations could have been generated at

Correspondence to: V. L. Krasovsky. the nonlinear stage of an electron beam-plasma instability. An essential prerequisite to such a hypothesis is also the strong correlation between broadband electrostatic noise and intensity of high-energy particle flows in the magnetotail (Gurnett et al., 1976; Grabbe and Eastman, 1984; Onsager et al., 1993; Kojima et al., 1994).

Many and varied numerical simulations on the behavior of electron beam-plasma systems (see e.g. the review by Berk and Roberts, 1970) show a comparatively fast linear stage of the instability accompanied by exponential growth of unstable plasma oscillations and nonlinear saturation process due to trapping of resonant particles. As a result of the beam trapping, a periodic sequence of electron bunches is formed. These bunches look like vortices in phase space and usually take the form of electron phase space density holes (EHs). Further evolution of the trapped electron bunches and corresponding nonlinear wave perturbations were observed in the numerical simulations of a long time dynamics of beam-plasma systems by Omura et al. (1996). It turned out that ESW associated with EHs are rather stable, so that the system evolves very slowly. Probably, the most bright phenomenon at this slow stage is coalescence and merging of the holes. The repeated EH coupling results in a generation of isolated nonlinear wave structures similar to those observed in space. Thus the numerical experiment exhibits a formation of the moving electrostatic pulses, suggesting that the corresponding generation mechanism can be treated as one of the most probable candidate to explain the ESW waveforms recorded by the Geotail spacecraft.

The coupling of EHs presents a mechanism of wave energy localization and growth of the ESW intensity. Therefore the questions of the dynamics of interacting EHs, as applied to the studies on ESW, take on fundamental significance. However, despite the repeated observations of the EH coupling by means of numerical simulation (see e.g. Berk et al., 1970; Ghizzo et al., 1988; Omura et al., 1996), physical reasons for the EH 
merging and character of ESW interaction are not entirely clear and call for an additional analysis. Theory of EH "collisions" is commonly restricted by estimates or qualitative reasonings (Berk et al., 1970; Dupree, 1982). A more extended and purposeful consideration of the ESW interaction is described in this paper. Analyzing the energy balance in a system of two interacting EHs, we will show that the irreversibility of the ESW interaction and inelastic nature of the EH collisions are explained by an effective heating of EHs caused by a violation of the adiabaticity of trapped electron motion and phase mixing process.

\section{ESW as a localized BGK perturbation}

To describe the interaction between ESWs we need first to choose an appropriate model of the wave perturbation under study. Since weakly interacting $\mathrm{EH}$ s evolve slowly in comparison with oscillations of trapped particles in the electrostatic potential wells, a single ESW may be treated as a localized stationary Bernstein-Greene-Kruskal (BGK) mode (Bernstein et al., 1957; Matsumoto et al., 1994; Krasovsky et al., 1997) or, more rigorously, as a slowly varying wave structure of the BGK type comoving with EH. Presenting a deficit of the trapped electrons, EH has a positive effective charge, negative mass and negative kinetic energy (Berk et al., 1970). The charge of the hole is shielded by background plasma electrons at some typical distance $\lambda$ dependent on the hole velocity $V_{0}$. At small $V_{0}$ the shielding length $\lambda$ is close to usual Debye length, while at high $V_{0}$, as is often the case in beam-plasma systems, $\lambda\left(V_{0}\right)$ may take diversified values depending on the electron distribution function in the vicinity $V_{0}$. In either case the plasma response to the perturbation of the electrostatic potential $\phi$ caused by EH can be described within the framework of the linear quasistatic approximation, as we deal with a weak wave perturbation slowly changing in the frame of reference moving together with EH. For brevity, the following units of measurement are in use below

$$
\begin{aligned}
& {[t]=\omega_{p}^{-1}, \quad[z]=\lambda_{D}, \quad[V]=\omega_{p} \lambda_{D} \equiv v_{t h},} \\
& {[n]=n_{0}, \quad[\phi]=m_{e} v_{t h}^{2} / e}
\end{aligned}
$$

for the time $t$, coordinate $z$ and other spatial scales, velocity $V$, densities $n$ and electrostatic potential $\phi$, where $n_{0}$ is the steady-state plasma density, $\lambda_{D}$ is the Debye length and $\omega_{p}$ is the electron plasma frequency. Then the corresponding quasistatic perturbation of the background electron density may be found with the aid of the simple expression $\tilde{n}=\phi / \lambda^{2}$, so that the Poisson equation in the comoving frame takes the form

$\frac{\partial^{2} \phi}{\partial z^{2}}=\frac{\phi}{\lambda^{2}}-n_{H}$ where $n_{H}$ is the density of the hole. Hereafter for the sake of convenience we set $n_{H}$, as well as other physical quantities relative to the hole, to be positive, taking into account the deficit of the trapped electrons in the hole with the help of the "minus" sign before the corresponding expressions for the distribution function, density, energy and effective mass of the hole.

Let $L$ be the size of $\mathrm{EH}$, so that $n_{H}>0$ at $|z|<L$, and $n_{H}=0$ elsewhere. Then outside of EH the solution of (1) describes just the exponential fall of the potential at infinity $|z| \rightarrow \infty$

$$
\phi(z)=\phi(L) \exp [(L-|z|) / \lambda] \quad, \quad|z| \geq L .
$$

In the interior of the hole $|z|<L$ the solution depends on its structure. A stationary BGK equilibrium exists provided the trapped particle distribution is a function of their energy in the wave frame $w=v^{2} / 2-\phi(z)$, i.e. $f_{H}(v, z)=f_{H}(w)$, satisfying the stationary Vlasov equation

$v \frac{\partial f_{H}}{\partial z}+\frac{\partial \phi}{\partial z} \frac{\partial f_{H}}{\partial v}=0$

Then the hole density is expressed by

$n_{H}(z)=2 \int_{-\phi(z)}^{-\phi(L)} \frac{d w f_{H}(w)}{\sqrt{2[w+\phi(z)]}}$.

For a given $f_{H}(w)$, the wave profile inside of EH may be found by substitution of (4) in (1) and solving the Poisson equation with the sewing together of the potential $\phi$ and its derivatives $\partial \phi / \partial z$ at the EH boundaries $z= \pm L$. Unfortunately, the choice of the trapped electron distribution is not unique, and one is thus forced to use one or another of artificial models for practical calculations. Since EH features a lack of the trapped electrons near the bottom of the potential trough (i.e. in the vicinity of the electrostatic potential maximum), the distribution $f_{H}(w)$, as an additive contribution to the total distribution function, should be taken to be monotonically increasing. The distributions of this kind lead to a bell-like potential profile, and the basic parameters of the BGK pulse are related by the following expressions

$$
\begin{aligned}
& \phi \sim \phi_{\max } \sim m \lambda, \quad \Delta v \sim m^{1 / 2} L^{1 / 2}, \\
& f_{H} \sim m^{1 / 2} L^{-3 / 2}
\end{aligned}
$$

where $\Delta v$ is the EH width in velocity space and by definition the $\mathrm{EH}$ mass equals to

$$
m=\iint d z d v f_{H}
$$

Finally, in the rest frame this "hump" of the electrostatic potential is moving together with $\mathrm{EH}$ and corresponds to a single ESW. 


\section{Analysis of ESW interaction process}

Consider an elementary event of interaction of two identical EHs moving with equal or very close velocities. Let the holes be originally spaced far apart, so that their interaction is weak owing to the shielding by plasma. Nevertheless they attract each other at any finite distance between them due to mutual disturbance of the self-consistent electrostatic potentials, as may be readily shown with the aid of the Vlasov-Poisson system of equations (see also a qualitative explanation given by Berk et al., 1970). Hence, the holes are gradually accelerated towards each other. However at the initial stage of the interaction, their positions, velocities and phase space structures are changing slowly in comparison with the oscillations of the trapped electrons in the self-consistent potential wells, as is also observed in the numerical experiments. This suggests the adiabatic approximation for a description of the motion of the trapped electrons comprising the holes and, thereby, an analytical consideration of the weak EH interaction. The corresponding technique has been developed recently for small EHs $L \ll \lambda$ on the basis of a consistent perturbation theory relative to the small parameter $\epsilon=L / \lambda \ll 1$, where the problem can be solved quite rigorously (Krasovsky et al., 1999). However, even in a more general case $\epsilon \leq 1$ the physical fundamentals of the $\mathrm{EH}$ interaction can be understood by means of a simple analysis of the energy conservation law. For brevity, below we will restrict ourselves by the discussion of the energy balance in the system of two interacting holes with an emphasis on the qualitative aspects of their dynamics.

The EH dynamics may be conveniently considered in the frame of reference comoving with their common center of mass. Owing to the symmetry of the problem on the interaction of the identical holes, the spatial dependencies of all physical quantities represent even or odd functions relative to the center of mass of the system $z=0$. In particular, the electrostatic potential $\phi(-z, t)=\phi(z, t)$ is an even function of the coordinate with zero derivative $\partial \phi / \partial z=0$ at $z=0$. Therefore, it will suffice to study the behavior of the system in one of the half-spaces, for example, in the right of them $z \geq 0$.

To derive the energy balance equation for the Vlasov fluid with the interacting EHs, the linear quasistatic approximation for the background electrons may be employed similarly to the previous section. Then the energy conservation law takes the form with the accepted accuracy

$$
\begin{gathered}
\int_{0}^{\infty} d z\left[(\partial \phi / \partial z)^{2}+(\phi / \lambda)^{2}\right]-m V_{c}^{2}- \\
\iint d z d v f_{H}\left(v-V_{c}\right)^{2}=i n v .
\end{gathered}
$$

where the first integral is the potential energy of the system $W_{p}$ representing the sum of the electrostatic ener- gy (the first term in square brackets) and perturbation of the background electron kinetic energy (the second term). The second contribution is the mechanical energy of the hole $W_{k}=m V_{c}^{2}>0$ caused by its motion as a whole with the velocity $V_{c}$ of its own center of mass $Z_{c}$. Finally, the last term is determined by the structure of the hole and corresponds to the internal hole energy $W_{i}$ associated with its finite sizes in phase space. For widely separated holes $Z_{c} \gg \lambda$, a comparison estimate of the contributions to (7) can be done with the help of the relations (5)

$W_{p} \sim W_{0}=m^{2} \lambda \quad, \quad W_{i} \sim m^{2} L$

The kinetic energy is considerably less than the potential energy $W_{k} \ll W_{p}$ at the initial stage of the interaction due to the small relative velocity of the holes. To a first approximation, the internal energy is negligible in accordance with (8), provided the $\mathrm{EH}$ size is small as compared to the shielding scale $\epsilon=L / \lambda \ll 1$. Then the dynamics of the system represents just usual oscillations of the two holes relative to their common center of mass owing to their mutual attraction and acceleration. Accelerating towards each other, the holes acquire kinetic energy $W_{k}$. The gain of the energy reaches the maximum $W_{k} \sim W_{0}$ when the holes meet with one another at the point of the common center of mass. The relative velocity becomes of the order of $V_{c} \sim m^{1 / 2} \lambda^{1 / 2}$, i.e. far above the size of the hole in velocity space $\Delta v$, as is seen from (5). As a consequence, the small holes cannot merge at their first intercrossing. However, a more careful analysis allowing for the higher order terms in $\epsilon$ shows that the hole internal energy $W_{i}$ grows on the average with every their passage through one another, so that an effective "friction" arises in the system, and the oscillations of the holes relative to the center of mass of the system become damping. The oscillating holes are gradually coming closer and closer together, and such a behavior serves as a precursor of the subsequent merging. It should also be emphasized that the potential energy of the system $W_{p}$ increases with the growth of $W_{k}$ due to the negativeness of the hole energy [see (7)]. In other words, the convergence of the holes is accompanied by the increase of the electrostatic energy of the order of $\Delta W_{p} \sim W_{0}$, and their merging must lead to some increment in $W_{p}$ as well.

Now let us consider the nature of the internal energy variations in the interaction process. The internal dynamics of a phase space "drop" of Vlasov fluid may be conveniently traced in the accompanying frame of reference comoving with its own center of mass $Z_{c}(t)$ : $\xi=z-Z_{c}(t), u=v-V_{c}(t)$. In this frame, the trapped electrons of the hole are oscillating in the effective potential well $\varphi(\xi, t)$ representing the sum of the electrostatic potential and potential of the inertia force. To determine the time variation of $W_{i}$, we need to find the trapped particle distribution function $f_{H}(\xi, u, t)$ 
through the Vlasov equation. Provided the time dependence of the effective potential is weak, the kinetic equation can be solved in the adiabatic approximation, where the distribution function is expressed in terms of the adiabatic invariant of the trapped particle motion

$f_{H}=f_{H}(J) \quad, \quad J=\oint d \xi u(\xi, w, t)$

where

$u= \pm \sqrt{2(w+\varphi)}$.

This expression simplifies the search for the self-consistent solution of the Vlasov-Poisson system of equations and, thereby, allows one to describe analytically the initial adiabatic stage of the $\mathrm{EH}$ acceleration. Omitting the corresponding mathematical treatment, here we note the characteristic features of the adiabatic stage. Except for small corrections to (9), a reversible (adiabatic) variation of the hole internal energy $W_{i}$ occurs at the initial stage of the interaction. Keeping watch on the time evolution of the hole structure in the phase space, one could see that the level curves of the distribution function hold their oval form and coincide approximately with the phase space trajectories of the trapped electrons much as they do in the case of BGK equilibria. The difference from exact stationary BGK equilibrium is that the trapped particle distribution function of $\mathrm{EH}$ depends, even though weakly, on time $f_{H} \simeq f_{H}(w, t)$, and the correction to this distribution $\tilde{f}(w, \xi, t)$ dependent on $\xi$ is nonzero, even if small.

As the holes are accelerating and coming closer together, the effective potential is varying more and more rapidly, and the adiabaticity of the trapped electron oscillations in the effective potential well is violated more and more perceptibly. The deviation from adiabaticity peaks during the "collision" of the holes in the vicinity of their common center of mass $z=0$, i.e. at their spatial overlapping, when $\left|Z_{c}\right| \leq L$. The corresponding disturbance of the hole distribution $f_{H}$ manifests itself in essential deviation of the distribution function level curves from the phase space trajectories of the trapped electrons. Such a distribution cannot be steady-state, and the trapped particle oscillations are reflected in rotation of the phase space volume mapping the hole. Furthermore, the frequency of the oscillations depends on the electron energy level $w$, so that individual elements of the hole phase volume are rotating with different angular velocities. As a consequence, the oscillatory motion of the trapped electrons is smoothing off the distribution function along their phase trajectories (see e.g. O'Neil, 1965). This is well-known phase mixing of trapped particles, in the process of which the distribution function level curves tip over and curl to an elongating spiral in the phase space. Simultaneously, the originally oval shape of the hole evolves into a phase space vortex. Therefore, the competition of disturbance of the $\mathrm{EH}$ distribution function and the phase mixing process leads to practically irreversible modifications of the hole structure and growth of the hole internal energy $W_{i}$ or, in other words, to an effective heating of the hole. This peculiar irreversibility is close in nature to the dynamical chaos arising in Hamilton systems (see e.g. Zaslavsky and Sagdeev, 1988), as the input VlasovPoisson equations do not contain any really dissipative terms.

Turning back to the energy balance equation (7), we note that at $\epsilon \ll 1$ the system of two interacting holes resembles a simple vibrating system with weak damping, while at $\epsilon \leq 1$ it is analogous to a system with strong damping. Mathematically, the problem on the interaction of pointlike holes $\epsilon \rightarrow 0$ is quite simple, though it is of little interest from physical point of view. At finite but small $\epsilon \ll 1$, the problem can be solved on the basis of a perturbation theory (Krasovsky et al., 1999). Finally, in the absence of small parameters an analytical consideration is extremely complicated. Nevertheless, the above mentioned physical mechanisms underlying the EH interaction and coupling refer equally to small $\epsilon$ as well as to large $\epsilon \simeq 1$. In the latter case the losses of the vibratory energy due to the irreversible growth of the hole internal energy become so high that the holes can coalesce and merge immediately in the course of their first "collision". The merging can be treated as a collectivization of the trapped electrons accompanied by their phase mixing. In order for this to happen, an electron must have time to execute one or several oscillations in the potential well during the spatial overlapping of the holes. By this is meant that the relative velocity of the holes must not exceed the oscillatory velocity of the trapped electrons. i.e. the typical size of the holes in the velocity space. The merging represents an essentially irreversible (nonadiabatic) process.

\section{Discussion and conclusions}

We have considered interaction of two isolated ESWs within the framework of the quasistatic description of plasma response to the slowly varying phase density EHs. In this section, we briefly review the mechanism of the ESW interaction, as applied to interpretation of the results of numerical studies on ESW generation.

In numerical simulations, as mentioned above, a periodic sequence of $\mathrm{EHs}$ arises at the saturation stage of a stream-driven instability. In that case the EH interaction may take a character of a slower secondary instability. Physical mechanism and consequences of the instability may be easily understood on the basis of the foregoing semiqualitative analysis. Since the holes attract each other, and the attractive force depends on the distance between them, a small displacement of the holes from their equilibrium positions would suffice to move the system off balance. As a result, oscillations of the holes relative to their equilibrium positions must 
arise. These oscillations must be damping owing to the irreversible losses of the vibratory energy of the system. Oscillating, the holes tend to unite by pairs into binary vibrating systems. The further time evolution of a pair of holes may considered analogously to the above analysis of the two hole system, as the influence of neighbouring pairs should be suppressed markedly by the background plasma shielding. Hence the physical meclianism of the instability and prerequisites for the subsequent ESW coupling become clear. Upon the ESW merging by pairs, this process can be multiply reproduced.

In summary we conclude that the violation of the adiabaticity of the trapped particle motion and phase mixing process lead to an effective heating of EHs and corresponding energy losses in the system of interacting ESW. This entails an irreversible in character and typical for dissipative systems behavior of the macroscopic: quantities describing the system. Such a dynamical irreversibility is an intrinsic property of the physical system under study and explains the general tendency to the EH coupling. The "collisions" of the phase density holes are always "inelastic", and the merging of the electrostatic solitary waves is natural and regular outcome of their interaction.

Acknowledgements. 'This work was initiated during the stay of one of the authors (V. L. K.) at liadio Atmospheric Scieuce Center, Kyoto University. He would like to thank Kyoto University for hospitality and the Ministry of Education, Science, Sports and Culture of Japan for financial support.

\section{References}

Berk, H. L. and Roberts, K. V., Water Bag Model, in Methods in Computational Physics, edited by B. Adler, S. Fernbach and M. Rotenberg, Academic Press, New York and London, Vol. 9, p. $87,1970$.

Berk, H. L., Nielsen C. N. and Roberts K. V., Phase space hydrodynamics of equivalent nonlinear systems: experimental and computational observations, Phys. Fluids, 13, 980-995, 1970.
Bernstein, I. B., Greene, J. M. and Kruskal, M. D., Exact nonlinear plasma oscillations, Phys. Rev., 108, 546-550, 1957.

Dupree, T. H., Theory of phase-space density holes, Phys. Fluids, 25, 277-289, 1982.

Ghizzo, A., Iznar, B., Bertraud, P., Fijalkov, F., Feix, M. R. and Shoucri, M., Stability of Bernstein-Greene-Kruskal plasma equilibria. Numerical experiments over a long time, Phys. Fluids, 31, 72-82, 1988.

Grabbe, C. L. and Eastman, T. E., Generation of broadband electrostatic noise by ion beam instabilities in the magnetotail, $J$. Geophys. Res., 89, 3865-3872, 1984.

Guruett, D. L., Frank, L. A. and Lepping, R., Plasma waves in the distant magnetotail, J. Geophys. Res., 81, 6059-6071, 1976.

Kojina, H., Matsumoto, H., Miyatake, T., Nagano, I., Fujita, A., Frank, L. A., Mukai, T., Paterson, W. R., Saito, Y., Machida, $S$. aud Anderson, R. R., Relation between electrostatic solitary waves and hot plasma flow in the plasma sheet boundary layer: GEOTAIL observations, Geophys. Res. Lett., 21, 2919-2922, 1994.

Krasovsky, V. L., Matsumoto, H. and Omura, Y., BernsteinGreene-Kruskal analysis of electrostatic solitary waves observed with Geotail, J. Geophys. Res., 102, 22,131-22,139, 1997.

Krasovsky, V.L., Matsumots, H. and Omura, Y., Interaction of small phase density holes, Physica Scripta, in press, 1999.

Matsumoto, H., Kojima, H., Miyatake, T., Omura, Y., Okada, M., Nagano, I. and Tsutsui, M., Electrostatic Solitary Waves (ESW) in the magnetotail: BEN wave forms observed by GEOTAIL, Geophys. Res. Lett., 21, 2915-2918, 1994.

Omura, Y., Kojizaa, H. and Matsumoto, H., Computer simulations of electrustatic solitary waves in the inaguetotail: A uonlinear model of broadband electrostatic noise, Geophys. Res. Lett., 21, 2923-2926, 1994.

Omura, Y., Matsumoto, H., Miyake, T. and Kojina, H., Electron beam instabilities as generation mechanism of electrostatic solitary waves in the magnetotail, J. Geophys. Res., 101, 2685-2697, 1996.

O'Neil, T. M., Collisionless damping of noulinear plasma oscillations, Phys. Fluids, 8, 2255-2262, 1965.

Onsager, T. G., Thomsen, M. F., Elphic, R. C., Gosling, J. T., Anderson, R. R. and Kettmann, G., Electron generation of electrostatic waves in the plasma sheet boundary layer, J. Geophys. Res., 98, 15,509-15,518, 1993.

Zaslavsky, G. M. and Sagdeev, R. Z., Introduction to Nonlinear Physics, Nauka, Moscow, 1988. 